\title{
ONE-DIMENSIONAL LONG-RANGE FERROMAGNETIC ISING MODEL UNDER WEAK AND SPARSE EXTERNAL FIELD
}

\author{
AZER KERIMOV \\ Department of Mathematics, Bilkent University, \\ 06800 Bilkent, Ankara, Turkey \\ kerimov@fen.bilkent.edu.tr
}

Received 5 March 2008

\begin{abstract}
We consider the one-dimensional ferromagnetic Ising model with very long range interaction under weak and sparse biased external field and prove that at sufficiently low temperatures, the model has a unique limiting Gibbs state.

Keywords: Ground state; Gibbs state; extreme Gibbs state; phase transition.

PACS numbers: $05.50 .+\mathrm{q}, 75.10 . \mathrm{Hk}$
\end{abstract}

\section{Introduction}

Consider the one-dimensional ferromagnetic Ising model with long range interaction:

$$
H_{0}(\phi)=-\sum_{x, y \in \mathbf{Z}^{1} ; x>y} U(x-y) \phi(x) \phi(y)
$$

where spin variables $\phi(x)$ associated with the one-dimensional lattice sites $x$ take values -1 and +1 and the pair potential $U(x-y)=(x-y)^{-\gamma}, 1<\gamma \leq 2$. The condition $\gamma>1$ is necessary for the existence of the thermodynamical limit. We are focused on the case $\gamma \leq 2$, otherwise $\sum_{x \in \mathbf{Z}^{1}, x>0} x U(x)<\infty$ and the model (1) has a unique Gibbs state. ${ }^{1-3}$

The low temperature phase diagram of the the model (1) was investigated in Refs. 4 and 5 for $1<\gamma<2$ and in Ref. 6 for the borderline case $\gamma=2$ : at all sufficiently large values of the inverse temperature, there exist at least two extremal Gibbs states $P^{+}$and $P^{-}$corresponding to the ground states $\phi=+1$ and $\phi=-1$. This delicate result is closely related to the phenomenon of the "surface tension" in one dimension. Other profound advances including results on the relation between Fortuin-Kasteleyn percolation and magnetization were obtained in borderline case $\gamma=2^{7,8}$ (for the detailed approach to the random cluster models see Refs. 9 and 10). An alternative approach to the investigation of phase diagrams of ferromagnetic 
systems based on detailed exploration of spin configurations geometry was given in Ref. 11 (for $1.5 \leq \gamma \leq 2$ ).

In this paper we investigate the phase diagram of the model (1) under additional external field:

$$
H(\phi)=H_{0}(\phi)+\sum_{x \in \mathbf{Z}^{1}} h_{x} \phi(x)
$$

Let $V_{N}$ be an interval with the center at the origin and with the length of $2 N$. The set of all configurations $\phi\left(V_{N}\right)$ we denote by $\Phi(N)$. The concatenation of the configurations $\phi\left(V_{N}\right)$ and $\phi^{i}\left(\mathbf{Z}^{1}-V_{N}\right)$ we denote by $\chi: \chi(x)=\phi(x)$, if $x \in V_{N}$ and $\chi(x)=\phi^{i}(x)$, if $x \in \mathbf{Z}^{1}-V_{N}$. Define

$$
H_{N}\left(\phi \mid \phi^{i}\right)=\sum_{B \subset \mathbf{Z}^{1}: B \cap V_{N} \neq \emptyset} U(\chi(B))
$$

The finite-volume Gibbs distribution corresponding to the boundary conditions $\phi^{i}$ is

$$
\mathbf{P}_{N}^{i}\left(\phi \mid \phi^{i}\right)=\frac{\exp \left(-\beta H_{N}\left(\phi \mid \phi^{i}\right)\right)}{\Xi\left(N, \phi^{i}\right)}
$$

where $\beta$ is the inverse temperature and the partition function $\Xi\left(N, \phi^{i}\right)=$ $\sum_{\phi \in V_{N}} \exp \left(-\beta H_{N}\left(\phi \mid \phi^{i}\right)\right)$. A probability measure $\mathbf{P}$ on the configuration space $\{-1,1\}^{\mathbf{Z}^{1}}$ is called an infinite-volume Gibbs state if for each $N$

$$
\mathbf{P}\left(\phi\left(V_{N}\right)=\varphi\left(V_{N}\right) \mid \phi\left(\mathbf{Z}^{1}-V_{N}\right)=\phi^{i}\left(\mathbf{Z}^{1}-V_{N}\right)\right)=\mathbf{P}_{N}^{i}\left(\varphi \mid \phi^{i}\right)
$$

for $\mathbf{P}$ almost all $\phi^{i}$ in $\{-1,1\}^{\mathbf{Z}^{1}}$.

Below we investigate the set of all infinite-volume Gibbs states of the model (2). As a matter of course, the sufficiently strong external field exterminates the longrange interaction and the dependence on the boundary conditions disappears when $N$ goes to infinity:

Theorem 1. At any fixed value of the inverse temperature $\beta$ there exists a constant $h_{0}$ such that for all realizations of the external field $\left\{h_{x}, x \in \mathbf{Z}^{1}\right\}$ satisfying $\left|h_{x}\right|>h_{0}, x \in \mathbf{Z}^{1}$ the model (2) has a unique infinite-volume Gibbs state.

The rigorous proof of this natural result follows from the following Theorem 2, treating a more general case which applies to a much wider class of interaction potentials. Consider a model on $\mathbf{Z}^{1}$ with the formal Hamiltonian

$$
H_{0}(\phi)=\sum_{B \subset \mathbf{Z}^{1}} U(\phi(B))
$$

where the spin variables $\phi(x) \in \Phi, \Phi$ is a finite subset of the real line $\mathbf{R}$, $\phi(B)$ denotes the restriction of the configuration $\phi$ to the set $B$, the potential $U(\phi(B))$ is not necessarily translationally invariant. On the potential $U(\phi(B))$ we impose a natural condition, necessary for the existence of the thermodynamic 
limit: $\sum_{B \subset \mathbf{Z}^{1}: x \in B}|U(\phi(B))|<C_{0}$, where the constant $C_{0}$ does not depend on $x$ and the configuration $\phi$. Consider a model with the Hamiltonian

$$
H(\phi)=H_{0}(\phi)+\sum_{x \in \mathbf{Z}^{1}} h_{x} \phi(x)
$$

where $\left\{h_{x}, x \in \mathbf{Z}^{1}\right\}$ is a random external field.

Theorem 2. ${ }^{12}$ For any model (3) and any fixed value of the inverse temperature $\beta$ there exists a constant $h_{0}$ such that for all realizations of the external field $\left\{h_{x}, x \in\right.$ $\left.\mathbf{Z}^{1}\right\}$ satisfying $\left|h_{x}\right|>h_{0}, x \in \mathbf{Z}^{1}$ the model (4) has a unique infinite-volume Gibbs state.

The model (2) has also a unique limiting Gibbs state when the value of the external field is small but the field is "very ordered": Consider a model (2) with periodic external field constituted by alternating $(+)$ and $(-)$ blocks:

$$
H(\phi)=-\sum_{x, y \in \mathbf{Z}^{1} ; x>y} U(x-y) \phi(x) \phi(y)+\sum_{x \in \mathbf{Z}^{1}} h_{x}^{r} \phi(x)
$$

where $h_{x}^{r}$ is a periodic function of period $2 r: h_{x}^{r}=h_{x+2 r k}$ for all integer values of $k$ and for some fixed positive $\epsilon$

$$
h_{x}^{r}= \begin{cases}+\epsilon & \text { if } x=1, \ldots, r \\ -\epsilon & x=r+1, \ldots, 2 r\end{cases}
$$

Theorem 3. ${ }^{13}$ Let $\epsilon$ be an arbitrary positive fixed number not exceeding some constant $h_{1}$. There exist natural numbers $R_{1}=R_{1}(\epsilon)$ and $R_{2}=R_{2}(\epsilon)$ such that at all sufficiently small temperatures the model (5) has at least two limiting Gibbs states for all $r \leq R_{1}$ and a unique infinite-volume Gibbs state for all $r>R_{2}$.

Most likely the values of $R_{1}$ and $R_{2}$ coincide, but the proof of this statement is unknown. For given $\epsilon$, the value of $R_{2}$ is chosen to be sufficiently large in order to provide the reduction of the influence of alternatively oriented neighbor blocks on the given block: the value of $R_{2}$ is greater than $N_{\epsilon}(M+1)$, where $2 \sum_{i=N_{\epsilon}+1}^{\infty} i^{-\gamma}<\epsilon$ and $M=\max \left(N_{1},(8 /(2-\gamma) \epsilon)^{\frac{1}{\gamma-1}}\right)$.

If the values of the external field at all lattice points are aligned, then the infinitevolume Gibbs state is unique ${ }^{14,15}$ at all values of the temperature. This result follows from the ferromagnetic nature of interaction and uses Fortuin-Ginibre-Kasteleyn or Griffiths-Hurst-Sherman inequalities.

In this paper we investigate the model (2) under small and sparse external biased field with changing signs. Let $h_{x}^{L}$ be a periodic function of period $3 L$ : for all integer values of $k$ and $n$

$$
h_{x}^{L}= \begin{cases}\epsilon & \text { if } x=3 k L \text { or } x=(3 k+1) L \\ -\epsilon & \text { if } x=(3 k+2) L \\ 0 & x \neq n L\end{cases}
$$

where $L$ is a positive constant and $0<\epsilon<U(1)$. 
Consider a model with the following Hamiltonian:

$$
H(\phi)=H_{0}(\phi)+\sum_{x \in \mathbf{Z}^{1}} h_{x}^{L} \phi(x)
$$

Theorem 4. For any values of the positive constants $\epsilon$ and $L$ at sufficiently small values of the temperature, the model (6) has a unique infinite-volume Gibbs state.

Since the additional nonsymmetric external field $h_{x}^{L}$ breaks the symmetry between $(+)$ and $(-)$ phases and leads to a unique ground state (unique zero temperature phase), the statement of Theorem 4 is physically to be expected. But in general the uniqueness of zero temperature phase can not guarantee the uniqueness at nonzero temperatures ${ }^{16}$ and the proof of Theorem 4 requires comparison of infinite-volume Gibbs states corresponding to different boundary conditions.

\section{Proof of Theorem 4}

In order to prove the uniqueness of Gibbs states, we use the method employing the close relationship between phase transitions and percolation in models with a unique ground state. ${ }^{17}$ The method uses the idea of "coupling" of two independent partition functions and is based on the method used in Ref. 18. Similar "coupling" arguments are also at the center of the disagreement percolation approach to Gibbs states uniqueness problem. ${ }^{19,20}$

Let $\mathbf{P}^{1}$ and $\mathbf{P}^{2}$ be two extreme limiting Gibbs states corresponding to the fixed boundary conditions $\phi^{1}$ and $\phi^{2}$. Since $\mathbf{P}^{1}$ and $\mathbf{P}^{2}$ are singular with respect to each other or coincide, ${ }^{21,22}$ in order to prove the uniqueness of the limiting Gibbs states of (6) we establish non-singularity of $\mathbf{P}^{1}$ and $\mathbf{P}^{2}$.

If the expression $\left|H_{N}\left(\phi \mid \phi^{i}\right)\right|$ expressing the energy of the configuration $\phi\left(V_{N}\right)$ at fixed boundary conditions $\phi^{i}\left(\mathbf{Z}^{1}-V_{N}\right)$ is bounded uniformly with respect to $N, \phi$ and $\phi^{i}$ then the non-singularity of $\mathbf{P}^{1}$ and $\mathbf{P}^{2}$ directly follows. This simple idea was firstly used in Ref. 3 for the proof of the absence of phase transition in one-dimensional models with long range interaction. But in our case $\left|H_{N}\left(\phi \mid \phi^{i}\right)\right|$ is not bounded and a more sophisticated approach is required.

Let $\phi_{N}^{\min }$ be the configuration with minimal energy at fixed $N$ and boundary conditions $\bar{\phi}$ :

$$
\min _{\phi \in \Phi(N)} H_{N}(\phi \mid \bar{\phi})=H_{N}\left(\phi_{N}^{\min , i} \mid \bar{\phi}\right)
$$

$H_{N}\left(\phi \mid \phi^{i}, \phi_{N}^{\min }\right)$ denotes the relative energy of a configuration $\phi$ (with respect to $\left.\phi_{N}^{\min }\right)$ :

$$
H_{N}\left(\phi \mid \phi^{i}, \phi_{N}^{\min }\right)=H_{N}\left(\phi \mid \phi^{i}\right)-H_{N}\left(\phi_{N}^{\min } \mid \phi^{i}\right) .
$$

Let $\mathbf{P}_{N}^{i}$ be Gibbs distributions on $\Phi(N)$ corresponding to the boundary conditions $\phi^{i}, i=1,2$ defined by the use of relative energies of configurations. Take $M<N$ and let $\mathbf{P}_{N}^{i}\left(\phi^{\prime}\left(V_{M}\right)\right.$ be the probability of the event that the restriction of the configuration $\phi\left(V_{N}\right)$ to $V_{M}$ coincides with $\phi^{\prime}\left(V_{M}\right)$. Based on the uniqueness of 
$\phi_{N}^{\min }$ we construct the contour model common for boundary conditions $\phi^{i}, i=1,2$ and by using of a well-known trick ${ }^{23}$ we come to "noninteracting" clusters from interacting contours (a cluster is a collection of contours connected by interaction bonds).

The cornerstone of the method is the estimation of the dependence of the expression $\mathbf{P}_{N}^{1}\left(\phi\left(V_{M}\right)\right) / \mathbf{P}_{N}^{2}\left(\phi\left(V_{M}\right)\right)$ on the boundary conditions $\phi^{1}$ and $\phi^{2}$ in terms of the sum of statistical weights of clusters connecting the cube $V_{M}$ with the boundary. At low temperatures, the application of this theory to one-dimensional models produces a uniqueness criterion ${ }^{17}$ which is formulated below as Theorem 5 .

A configuration $\phi^{g r}$ is said to be a ground state of the model (2), if for any finite set $A \subset \mathbf{Z}^{1} H\left(\phi^{\prime}\right)-H\left(\phi^{g r}\right) \geq 0$, where $\phi^{\prime}$ is a perturbation of $\phi^{g r}$ on the set $A$. We say that the ground state $\phi^{g r}$ satisfies the Peierls stability condition with a positive constant $\tau$ if for any finite set $A \subset \mathbf{Z}^{1} H\left(\phi^{\prime}\right)-H\left(\phi^{g r}\right) \geq \tau|A|$, where $|A|$ denotes the number of sites of $A$ and $\phi^{\prime}$ is a perturbation of $\phi^{g r}$ on the set $A$.

Condition 1. The model has a unique ground state satisfying the Peierls stability condition.

Condition 2. A constant $\alpha<1$ exists such that for any number $L$ and any interval $I=[a, b]$ with the length $n$ and for any configuration $\phi(I)$

$$
\sum_{B \subset \mathbf{Z}^{1} ; B \cap I \neq \emptyset, B \cap\left(Z^{1}-[a-L, b+L]\right) \neq \emptyset}|U(B)| \leq \text { const } n^{\alpha} L^{\alpha-1} .
$$

The Condition 2 is very natural and obviously is held for a pair potential $U(x-y)=$ $(x-y)^{-\gamma}(1<\gamma \leq 2)$ of the model $(6)$.

Theorem 5. ${ }^{17}$ Suppose that a one-dimensional model with a finite spin space and with the translationally-invariant Hamiltonian

$$
H(\phi)=\sum_{B \subset \mathbf{Z}^{1}} U(\phi(B))
$$

where $\sum_{B \subset \mathbf{Z}^{\mathbf{1}} ; x \in B}|U(B)|<\mathrm{const}$, satisfies the Conditions 1 and 2. Then a value of the inverse temperature $\beta_{1}$ exists such that if $\beta>\beta_{1}$ then the model has a unique limiting Gibbs state.

We can treat the model (6) as a translationally invariant model: if we partition the lattice into disjoint intervals $[3 k L, 3(k+1) L-1]$ and replace the spin space $\{1,-1\}$ by $\{1,-1\}^{[0,3 L-1]}$ including $2^{3 L}$ elements, then the model from translationally periodic with period $L$ transfers to translationally invariant model. Thus, for employing of Theorem 5 we have to control the validity of Condition 1 .

Lemma 1. The constant configuration $\phi^{+}=+1$ is a ground state of the model (6) and this configuration satisfies the Peierls stability condition. 
Proof. Let $\phi^{\prime}$ be a perturbation of $\phi^{+}$on the finite set $A$. A point $x \in \mathbf{Z}^{1}$ is said to be non regular if $\phi(x)=-1$. Two non regular point are connected if all points between them are non regular. The connected components of all non regular points we call contours. All nonzero interaction terms $U(x-y)(1-\phi(x) \phi(y))$ of $H\left(\phi^{\prime}\right)-H\left(\phi^{+}\right)$ are nonnegative. We take into account only terms $U(1)(1-\phi(x) \phi(y))$ representing interaction between neighboring spins, terms $h_{x}^{L}\left(\phi(x)-\phi^{+}(x)\right)$ and ignore all other positive interaction terms. Suppose that the set $A$ includes $n$ contours $K_{1}, \ldots, K_{n}$. Then

$$
\begin{aligned}
H\left(\phi^{\prime}\right)-H\left(\phi^{+}\right) & \geq \sum_{i=1}^{n}\left(\sum_{x \text { or } y \in K_{i}} U(1)(1-\phi(x) \phi(y))+\sum_{x \in K_{i}} 2 h_{x}^{L}\right) \\
& \equiv \sum_{i=1}^{n} \Delta\left(K_{i}\right) .
\end{aligned}
$$

Now we note if $K_{i}$ contains $n_{i}$ sites with $h_{x}=-\epsilon$, then $\Delta\left(K_{i}\right)$ includes exactly two $2 U(1)$ terms and at least $2\left(n_{i}-1\right)$ terms $2 \epsilon$ and the length of $K_{i}$ is at most $3 L n_{i}$. Consequently,

$$
\sum_{x \text { or } y \in K_{i}} U(1)(1-\phi(x) \phi(y))+\sum_{x \in K_{i}} 2 h_{x}^{L} \geq 2 U(1)-2 \epsilon+2 \epsilon\left(n_{i}-1\right)
$$

and the inequality $H\left(\phi^{\prime}\right)-H\left(\phi^{g r}\right) \geq \tau|A|$ holds with

$$
\tau=\min \left\{\frac{2 U(1)-2 \epsilon}{3 L}, \frac{2 \epsilon}{3 L}\right\}
$$

Lemma 1 is proved.

Lemma 2. The configuration $\phi^{+}=+1$ is a unique ground state of the model $(6)$.

Proof. Let $\phi^{g r}$ be a ground state of the model (6) and $\phi^{g r}=\phi^{+}$. We divide the proof into four cases.

Case 1. The total number of sites with $\phi^{g r}(x)=-1$ is finite. We get contradiction with Lemma 1 , since now $\phi^{g r}$ can be treated as a finite perturbation of $\phi^{+}$.

Case 2. The total number of sites with $\phi^{g r}(x)=+1$ is finite. For each natural $n$ we can find an interval $I_{k, n}=[3 k L,(3(k+n)+2) L]$ such that $k$ is an integer and $\phi^{g r}(x)=-1$ for each $x \in I_{k, n}$. Consider a finite perturbation $\phi^{\prime}$ of $\phi^{g r}$ on $A=I_{k, n}$. Then for sufficiently large values of $n$

$$
H\left(\phi^{\prime}\right)-H\left(\phi^{g r}\right) \geq 2 \epsilon(n+1)-2 \sum_{x \in I_{n}, y \notin I_{n}}|x-y| U(|x-y|)>0
$$

since $\left.\sum_{x \in I_{k, n}, y \notin I_{k, n}}|x-y| U(|x-y|)=\sum_{x \in I_{k, n}, y \notin I_{k, n}}|x-y|^{1+\gamma}\right) \leq$ const . $((3 n+2) L)^{2-\gamma}$ and $2-\gamma<1$. 
Case 3. The total number of sites with $\phi^{g r}(x)=+1$ and $\phi^{g r}(x)=-1$ is infinite and there is a finite interval $[-M, M]$ such that both configurations $\phi^{g r}(-\infty,-M)$ and $\phi^{g r}(M, \infty)$ are constant configurations. Without loss of generality, suppose that $\phi^{g r}(x)=-1$ for each $x \in(-\infty,-M)$. Then for arbitrary natural $n$, there exists a natural number $k$ such that the interval $I_{k, n}=[-3 k L,(3(k+n)+2) L]$ is a subset of $(-\infty,-M)$. Consider a finite perturbation $\phi^{\prime}$ of $\phi^{g r}$ on $A=I_{k, n}$. Then for sufficiently large values of $n$

$$
H\left(\phi^{\prime}\right)-H\left(\phi^{g r}\right) \geq 2 \epsilon(n+1)-2 \sum_{x \in I_{k, n}, y \notin I_{k, n}}|x-y| U(|x-y|)>0
$$

since $\left.\sum_{x \in I_{k, n}, y \notin I_{k, n}}|x-y| U(|x-y|)=\sum_{x \in I_{k}, y \notin I_{k, n}}|x-y|^{1+\gamma}\right) \leq$ const . $((3 n+2) L)^{2-\gamma}$ and $2-\gamma<1$.

Case 4. The total number of sites with $\phi^{g r}(x)=+1$ and $\phi^{g r}(x)=-1$ is infinite and for each natural number $n$ there exists an interval $I_{n}=\left[a_{n}, b_{n}\right]$ such that $\phi^{g r}\left(a_{n}\right)=\phi^{g r}\left(b_{n}\right)=1$ and the number of sites $x$ in $I_{n}$ with $\phi^{g r}(x)=-1$ is at least $n$. Consider a finite perturbation $\phi^{\prime}$ of $\phi^{g r}$ on $A$, where $A$ is the set of all sites in $I_{n}$ with $\phi^{g r}(x)=-1$. Then by Lemma 1

$$
\begin{aligned}
H\left(\phi^{\prime}\right)-H\left(\phi^{g r}\right) & \geq \tau n-\sum_{x \in A, y \notin I_{n}}|x-y| U(|x-y|) \geq \tau n-|A|^{2-\gamma} \\
& =\tau n-\mathrm{const} \cdot|n|^{2-\gamma}>0
\end{aligned}
$$

for sufficiently large values of $n$.

Lemma 2 is proved.

Lemmas 1 and 2 provide that the model (6) satisfies the Condition 1 and Theorem 4 follows from Theorem 5 .

\section{Concluding Remarks}

Theorem 4 has a straightforward generalization to any external field under which the model (2) has a unique ground state satisfying the Peierls stability condition. We expect that Theorem 4 holds for all periodic external fields with period $L$ satisfying $\sum_{x=0}^{L-1} h_{x} \neq 0$. Let us define a configuration $\varphi_{h}$ by $\varphi_{h}(x)=\operatorname{sign}\left(h_{x}\right)$. The ground state of the model (2) revealing as a result of ferromagnetical "struggle" between spins of $\varphi_{h}$ for some realizations of the external field is not unique or does not satisfy the Peierls condition and we can not prove Theorem 4 by applying the results of Ref. 17. We think that in these extreme cases some technical modifications of methods of Ref. 17 will lead to the proof of Theorem 4.

The ferromagnetical nature of interaction is not essential for the methods of the present paper and was used only for description of ground states.

The Peierls condition is essential for the absence of phase transitions (the fact that a one-dimensional model with translationally-invariant long-range interaction 
has a unique ground state can not guarantee the uniqueness of infinite-volume Gibbs states $\left.^{16}\right)$.

\section{Acknowledgments}

The author thanks the referees for their valuable comments and suggestions.

\section{References}

1. R. L. Dobrushin, Theor. Prob. Appl. 18, 201 (1968).

2. R. L. Dobrushin, Func. Anal. Appl. 2, 44 (1968).

3. D. Ruelle, Comm. Math. Phys. 9, 267 (1968).

4. F. Dyson, Comm. Math. Phys. 12, 91 (1969).

5. F. Dyson, Comm. Math. Phys. 21, 269 (1971).

6. J. Frohlich and T. Spencer, Comm. Math. Phys. 84, 91 (1982).

7. M. Aizenman and C. M. Newman, Comm. Math. Phys. 107, 611 (1986).

8. M. Aizenman, J. T. Chayes, L. Chayes and C. M. Newman, J. Stat. Phys. 50, 1 (1988).

9. H.-O. Georgii, O. Häggström and C. Maes, Phase Transitions and Critical Phenomena, Vol. 18 (Academic Press, 2001), p. 1.

10. G. Grimmett, The Random Cluster Model (Springer, Berlin, Heidelberg, Germany, 2006).

11. M. Cassandro, P. A. Ferrari, I. Merola and E. Presutti, J. Math. Phys. 46, 053305 (2005).

12. A. Kerimov, Int. J. Mod Phys. B 17, 5781 (2003).

13. A. Kerimov, J. Phys. A 40, 10407 (2007).

14. J. L. Lebowitz and A. Martin-Löf, Comm. Math. Phys. 25, 276 (1972).

15. C. J. Preston, Comm. Math. Phys. 35, 253 (1974).

16. M. Biskup, L. Chayes and N. Crawford, J. Stat. Phys. 122, 1139 (2006).

17. A. Kerimov, J. Phys. A 35, 5365 (2002).

18. A. Kerimov, J. Stat. Phys. 72, 571 (1993).

19. J. Van den Berg, Comm. Math. Phys. 152, 161 (1993).

20. J. Van den Berg and C. Maes, Ann. Prob. 22, 749 (1994).

21. Ya. G. Sinai, Theory of Phase Transitions: Rigorous Results (Pergamon Press, Oxford, 1982).

22. H.-O. Georgii, Gibbs Measures and Phase Transitions (De Gruyter, Berlin, 1988).

23. J. Bricmont, K. Kuroda and J. L. Lebowitz, Comm. Math. Phys. 101, 501 (1985). 